\title{
Functional gene screening in embryonic stem cells implicates Wnt antagonism in neural differentiation
}

\author{
Jerôme Aubert, Hannah Dunstan, Ian Chambers, and Austin Smith*
}

Published online 25 November 2002; doi:10.1038/nbt763

\begin{abstract}
The multilineage differentiation capacity of mouse embryonic stem (ES) cells offers a potential testing platform for gene products that mediate mammalian lineage determination and cellular specialization. Identification of such differentiation regulators is crucial to harnessing ES cells for pharmaceutical discovery and cell therapy. Here we describe the use of episomal expression technology for functional evaluation of cDNA clones during ES-cell differentiation in vitro. Several candidate cDNAs identified by subtractive cloning and expression profiling were introduced into ES cells in episomal expression constructs. Subsequent differentiation revealed that the Wnt antagonist Sfrp2 stimulates production of neural progenitors. The significance of this observation was substantiated by forced expression of Wnt- 1 and treatment with lithium chloride, both of which inhibit neural differentiation. These findings reveal the importance of Wnt signaling in regulating ES-cell lineage diversification. More generally, this study establishes a path for rapid and direct validation of candidate genes in ES cells.
\end{abstract}

Mouse embryonic stem (ES) cells undergo unlimited self-renewal in the presence of the cytokine leukemia inhibitory factor (LIF) while retaining multilineage differentiation capacity ${ }^{1}$. Withdrawal of LIF and aggregation leads to differentiation of structures known as embryoid bodies ${ }^{2}$. Embryoid body differentiation reproduces many aspects of tissue formation during the crucial but least accessible stages of mammalian embryogenesis shortly after implantation ${ }^{3}$. This provides an attractive system for the discovery of genes induced during early development and for validation of function in lineage specification, commitment, and differentiation. Such investigations are crucial for the prospects of controlling human pluripotent stem cell differentiation for biomedical applications ${ }^{4}$.

ES cells give rise to neural lineages following formation of embryoid bodies and treatment with all-trans retinoic acid (RA $)^{5-8}$. The mechanism by which RA exerts this effect is obscure. We sought to identify genes that could play a role in the commitment or differentiation of the neural lineage. We generated a subtractive library enriched for genes induced in RA-treated embryoid bodies. cDNAs of interest, defined by sequence analysis, were examined for neural-restricted expression in vitro and in the mouse embryo. Candidates were then functionally tested by introduction into ES cells using an episomal vector system to facilitate transfection and cDNA expression. Through this screening, we identified the secreted Wnt antagonist Sfrp2 (secreted frizzled-related protein-2) as a modulator of neural differentiation from ES cells.

\section{Results}

Subtractive cDNA screen. Expression of Sry-related high mobility group (HMG)-box transcription factor Sox1 (ref. 9) was analyzed to monitor neural differentiation in embryoid bodies. Sox1 expression is activated in the neural plate and subsequently maintained specifically in dividing neural precursor cells in the mouse embryo ${ }^{10}$. After RA treatment, Sox 1 mRNA was strongly induced in embryoid bodies. In contrast, Oct4 (also known as Pou5f1) mRNA, an essential marker for undifferentiated ES cells ${ }^{11}$, was downregulated. Without RA treatment, Sox 1 expression was not detectable, and Oct4 mRNA was reduced but not eliminated, reflecting nonneural differentiation with persistence of a subpopulation of undifferentiated ES cells. On the basis of these results, RNA samples from the end of day 8 were taken for CDNA preparation.

Normalized forward (+RA) and reverse (-RA) subtractive cDNA populations were generated using the PCR suppression subtractive hybridization ( $\mathrm{SSH}$ ) technique ${ }^{12}$. The forward subtractive cDNA was used to prepare a library potentially enriched for genes induced during neural determination. An aliquot of this library was plated, and 480 colonies containing inserts were isolated. The inserts were amplified and arrayed in duplicated sets of 96 double spots on nylon membranes. The membranes were then screened by differential hybridization with forward or reverse subtracted cDNA (Fig. 1). Clones that hybridized specifically to the forward probe were sequenced. After redundant clones had been eliminated, 96 unique sequences remained (see Supplementary Table 1 online). BLAST analyses indicated that 74 clones showed near or identical matches to known genes, 21 were expressed sequence tags (ESTs) mapping to separate UniGene clusters, and 1 showed no match. Of the known genes, no expression data are available for 23, whereas 37 (50\%) have been reported in developing or adult CNS. Of the remainder, 2 genes are directly responsive to RA but not neural-restricted, and $12(11 \%)$ have widespread expression or have been described before only in nonneural tissues. 


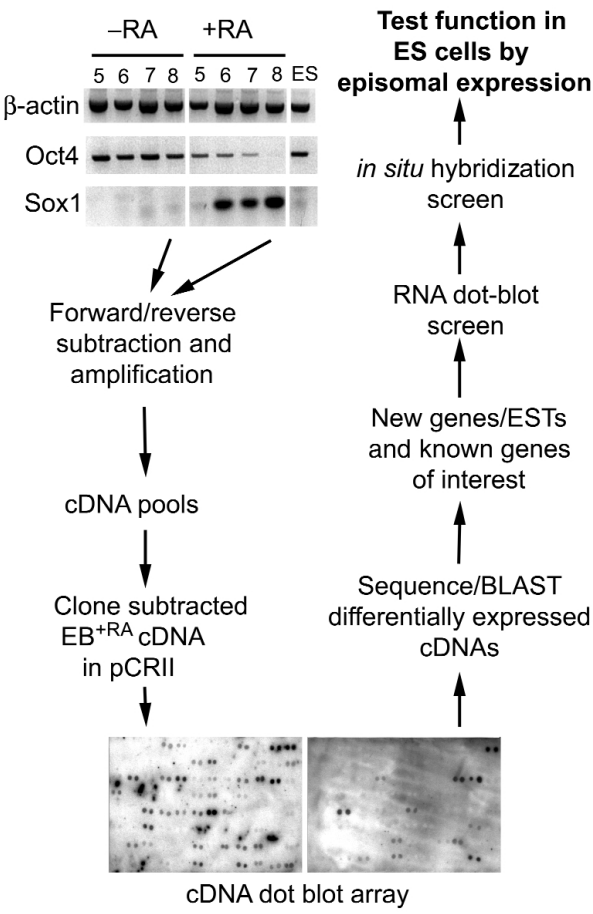

Confirmation of differential expression by RNA dot blot. Dot blots were prepared with RNA extracted from ES cells, embryoid bodies, neuroepithelial progenitor cells, and adult brain. We used Sox2- $\beta$-geo lineage selection ${ }^{8,13}$ to purify neuroepithelial progenitor cells from embryoid body derivatives. Blots were hybridized with probes prepared from individual cloned inserts and normalized relative to $\beta$-actin. We eliminated a small number of false-positive clones that failed to show differential hybridization and clones that appeared to be directly induced by RA rather than specific to neural precursors. Table 1 details the genes or ESTs that exhibit high expression in neural progenitors. Several genes earlier reported to be expressed in the embryonic or fetal CNS, such as Meis2 (ref. 14), Sfrp2 (ref. 15), Zic1 (ref. 16), Sox11 (refs 17, 18), Sox4 (ref. 19), Opr (ref. 20), and $m t s h 1$ (ref. 21), showed substantial induction during neural determination of ES cells and were downregulated in differentiated neurons and glia represented by adult brain. In addition, genes such as Tera and fidgetin ${ }^{22}$ were identified that are expressed appreciably in ES cells and maintained in neural progenitors but downregulated during nonneural differentiation.

Expression in the developing nervous system. The likely involvement of particular genes in neural development was then assessed by whole-mount in situ hybridization of mouse embryos. We found that genes of hitherto uncharacterized embryonic expression, Limd1 (ref. 23), Tera, RIKEN cDNA 2810027019 , and the mouse homolog of Sip1 (ref. 24), are expressed in the developing CNS. By way of example, data on three genes are presented in Figure 2. Tera, originally isolated from embryonal carcinoma cells, and P311, previously reported in adult brain regions and in neurons in striatum and superficial cortical

\begin{tabular}{lccccc}
\hline Table 1. RNA dot-blot expression screen & a & & & & \\
\hline & $\mathbf{4 - / 4 +}$ & $\mathbf{4 - / 4}$ & ES cells & Sox2 cells $^{+}$ & Brain \\
\hline UniGene cluster Mm.22383 & 1.00 & 0.50 & 1.30 & 1.25 & 0.90 \\
Tera & 1.00 & 0.80 & 1.10 & 1.60 & 0.00 \\
Fidgetin & 1.00 & 0.60 & 1.10 & 0.90 & 0.00 \\
RIKEN cDNA 2610510E10 (mSip-1) & 1.00 & 0.55 & 0.80 & 0.80 & 0.60 \\
FLJ20003 & 1.00 & 0.50 & 0.60 & 2.00 & 0.40 \\
IDN3 & 1.00 & 0.65 & 0.50 & 1.80 & 0.80 \\
UniGene cluster Mm.153458 & 1.00 & 0.35 & 0.50 & 1.60 & 1.50 \\
APP-BP1 & 1.00 & 0.50 & 0.50 & 1.30 & 0.20 \\
LNR42 & 1.00 & 0.50 & 0.50 & 1.30 & 0.20 \\
RIKEN cDNA 1200003107 & 1.00 & 0.30 & 0.40 & 1.50 & 0.65 \\
Limd1 & 1.00 & 0.25 & 0.40 & 1.50 & 0.10 \\
Sox11 & 1.00 & 0.40 & 0.40 & 1.30 & 0.75 \\
TCFL5 & 1.00 & 0.35 & 0.40 & 1.10 & 0.50 \\
UniGene cluster Mm.43640 & 1.00 & 0.45 & 0.30 & 2.00 & 1.50 \\
Sfrp2 & 1.00 & 0.40 & 0.30 & 0.95 & 0.10 \\
D21 & 1.00 & 0.40 & 0.30 & 0.90 & 0.30 \\
Zic1 & 1.00 & 0.40 & 0.20 & 1.50 & 0.90 \\
Sox4 & 1.00 & 0.20 & 0.15 & 1.50 & 0.10 \\
mtsh1 & 1.00 & 0.20 & 0.15 & 1.45 & 0.10 \\
Septin-6 & 1.00 & 0.30 & 0.15 & 1.00 & 0.35 \\
RIKEN cDNA 2810027019 & 1.00 & 0.25 & 0.10 & 3.00 & 0.30 \\
Meis2 & 1.00 & 0.20 & 0.10 & 2.00 & 0.40 \\
P311 & 1.00 & 0.20 & 0.10 & 1.30 & 0.30 \\
opr & 1.00 & 0.40 & 0.00 & 1.80 & 1.50 \\
UniGene cluster Mm.203935 & 1.00 & 0.40 & 0.00 & 1.20 & 0.90 \\
\hline
\end{tabular}

${ }^{a}$ Dot blots were probed with inserts from individual SSH clones, and the signals were quantitated by Phosphorlmage analysis and normalized relative to a $\beta$-actin control. Signals are expressed as a fraction of that obtained from RA-treated embryoid bodies. RNAs are from embryoid bodies treated with RA (4-/+), embryoid bodies without RA treatment (4-/4-), ES cells, Sox2-selected neural precursor cells, and adult brain.
Figure 1. Experimental overview. Total RNAs from undifferentiated ES cells and from embryoid bodies from days 5 to 8 were analyzed by RT-PCR. Subtracted cDNA from day 8 embryoid bodies was then taken through the outlined screening protocol.

layers during late gestation ${ }^{25}$, are both of unknown function. Sfrp2 encodes an extracellular Wnt antagonist ${ }^{26}$. All three genes are expressed along the length of the neural tube and in the head folds in mouse embryos at embryonic day (E) 8.5, but are absent from other embryonic lineages. Expression is maintained along the entire anteroposterior axis of the neural tube at E10.5, although regional restriction becomes evident in the brain (not shown). In transverse sections through the neural tube at E10.5, hybridization to P311 and Tera mRNAs is restricted dorsally, whereas $S f r p 2$ transcripts are localized medially. At this stage, $P 311$ transcripts are also detected in the dorsal dermamyotome, the sclerotome, and the apical ectodermal ridge, and Sfrp2 mRNA appears beyond the CNS in two longitudinal stripes corresponding to the mesonephros, as reported earlier ${ }^{15}$.

Functional analysis in ES-cell differentiation. Through the preceding screening steps, we focused on $11 \mathrm{cDNAs}$ of potential interest. Among these, 5 (P311, Tera, Limd1, LNR42, and RIKEN cDNA 2810027019) encode unknown proteins. In addition, we considered cDNAs encoding four transcription factors (Meis2, Mtsh1, Zic1, Sox11), a splicing factor (Sip1), and the Wnt antagonist Sfrp2. Open reading frames (ORFs) were generated by PCR to test the functionality of these genes during neural specification and differentiation.

Our approach was to introduce these cDNAs in parallel into ES cells and to assay their effects on differentiation. To achieve this, we harnessed the polyoma virus DNA replication system for episomal propagation of cDNA expression constructs. Expression of polyoma virus large $\mathrm{T}$ protein in recipient ES cells allows extrachromosomal replication of transfected DNA vectors that contain the polyoma ori- 


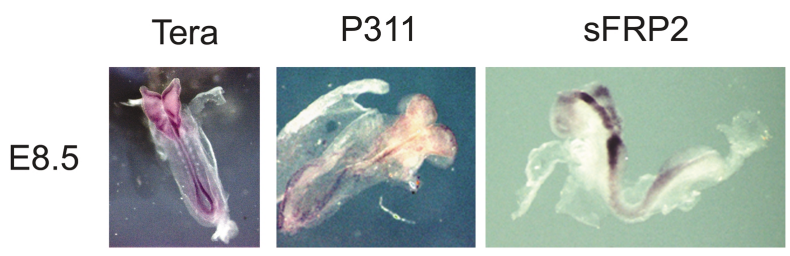

E10.5
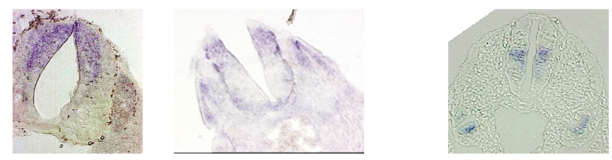

Figure 2. Expression of selected cDNAs in the embryonic CNS. Embryos at E8.5 and E10.5 were analyzed by whole-mount in situ hybridization using probes for the indicated mRNAs. For E10.5, transverse sections are shown through the neural tube.

gin of replication ${ }^{27}$. Under these conditions, 100 -fold more stable transfectants are obtained than by conventional transfection protocols that rely on chromosomal integration. This process is termed supertransfection ${ }^{27,28}$. We have developed this method for efficient cDNA transduction and expression in ES cells ${ }^{29,30}$. Provided that selection is maintained, an introduced Ori-containing plasmid is retained extrachromosomally in several copies per cell in both undifferentiated E14/T ES cells and differentiating progeny. An additional attribute of episomal supertransfection is that the introduced cDNAs avoid silencing, position effect variegation, and other unpredictable effects on level and specificity of expression that compromise chromosomally integrated plasmid or viral vectors.

The ORFs were inserted into the pPyCAGIP bicistronic supertransfection vector ${ }^{30}$. After electroporation and selection in puromycin, no colonies were obtained expressing Sip1, indicative of a toxic effect, and only small colonies that contained mainly differentiated cells were obtained for Meis2A, Sox 11, and Zicl, suggesting that overexpression of these factors compromises ES-cell viability or self-renewal. For the remaining seven cDNAs, large numbers of undifferentiated ES-cell colonies were obtained, comparable to those generated with an enhanced green fluorescence protein (eGFP) control vector. Colonies $(\sim 500)$ were pooled by trypsinization and polyclonal transfectant populations established.

The functional consequence of forced expression of these cDNAs was then examined by embryoid body differentiation with and without RA treatment. For six of the cDNAs, there were no overt differences in the differentiation profile from control transfectants. In contrast, cells expressing the Wnt antagonist Sfrp2 showed markedly enhanced neuronal differentiation in response to RA. Yet more strikingly, Sfrp2 transfectants produced appreciable numbers of neurons without RA treatment (Fig. 3). Expression of Sfrp2 induces the emergence of cells with extended processes that are immunoreactive for the neuronal marker class III $\beta$-tubulin $\left(\mathrm{TuJ}^{+}\right.$cells). Genes expressed in neural precursors, such as Ngn2 (ref. 31), Pax6 (ref. 32), and $P a x 7$ (ref. 33), are upregulated along with the neuronal gene $S h c C$ (ref. 34). These data show that forced expression of Sfrp2 promotes neural and neuronal gene expression and the appearance of morphologically differentiated neurons.

Induction of neural precursors by forced expression of Sfrp2. To obtain independent confirmation of and further insight into the effect of Sfrp2 on neural differentiation, we took advantage of an ES-cell line engineered to monitor neural specification. In 46C ES cells, a Sox1 reporter allele has been created by replacing the ORF of the Sox 1 gene with the coding sequence for eGFP. This GFP knockin is silent in undifferentiated ES cells but is activated early in neural specification, just as is endogenous Sox1 (ref. 10). The kinetics and frequency of conversion of ES cells to neural precursors can therefore be monitored and quantitated by FACS analysis of GFP fluorescence.

46C ES cells do not express polyoma large T. Therefore, conventional stable integrants were generated by electroporation of linearized pPyCAG-Sfrp-IP. The bicistronic selection cassette permits enrichment for colonies that exhibit robust expression of the inserted cDNA. Puromycin-resistant colonies (10-15) were pooled to establish a polyclonal population that expresses Sfrp2 (Fig. 4). Embryoid bodies were then formed from these transfectants. FACS analyses presented in Figure 4 show that Sfrp2 expression allows development of Sox1-GFP ${ }^{+}$cells without exposure to RA. The frequency of these cells is $30-40 \%$ by day 8 , which is comparable to that obtained by RA treatment of the untransfected 46C cells. Furthermore, as with E14/T supertransfection, Sfrp2 expression in $46 \mathrm{C}$ cells enhances the neural-inducing effect of RA such that $>60 \%$ of cells are Sox $1-\mathrm{GFP}^{+}$by day 8 .

Wnt activity inhibits neural differentiation. Sfrp2 acts as an extracellular antagonist of Wnts by competing with transmembrane frizzled receptors ${ }^{35-37}$. The stimulatory effect of Sfrp2 on neural differentiation therefore implies that Wnt activity is inhibitory to the neural program. To examine this directly, we introduced a Wnt1 expression vector into E14/T cells. The capacity of Wnt-1-overexpressing cells to produce neurons after RA treatment of embryoid bodies was compared with that of control transfectants. A greatly reduced number of $\mathrm{TuJ}^{+}$neurons were evident (Fig. 5). The expression of neural and neuronal marker genes was also diminished in Wnt-1-expressing cells. We also investi-

A

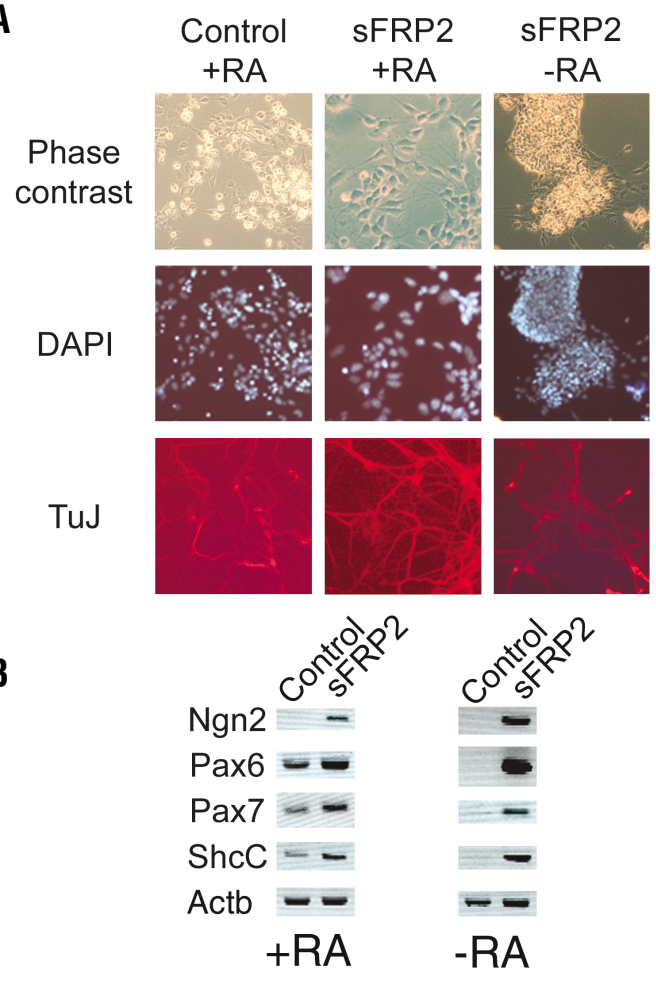

Figure 3. Effect of Sfrp2 expression in E14/T cells during ES cell differentiation. (A,B) Embryoid bodies formed from GFP control or Sfrp2 transfectants were stimulated from day 5 to day 8 with $10^{-6} \mathrm{M}$ RA or vehicle $(0.1 \%$ dimethyl sulfoxide, DMSO). After dissociation and plating at day 8 , cells were cultured for a further four days. RNA was then extracted for RT-PCR analysis (B) or cells fixed for immunostaining with anti-class III- $\beta$ tubulin (TuJ) (A). No TuJ signal was obtained from control cells without RA treatment. 
A

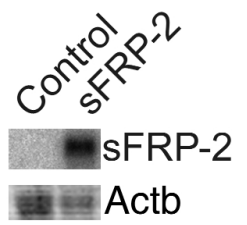

Northern blot

B
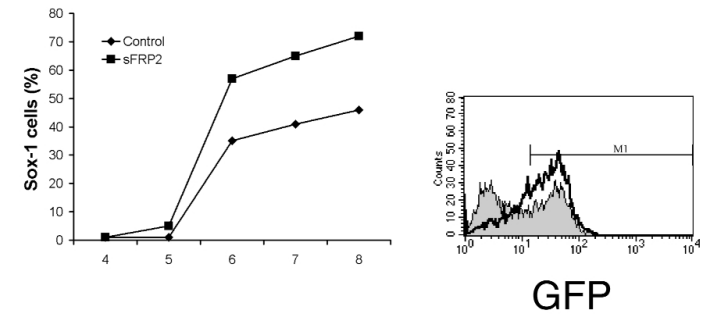

C
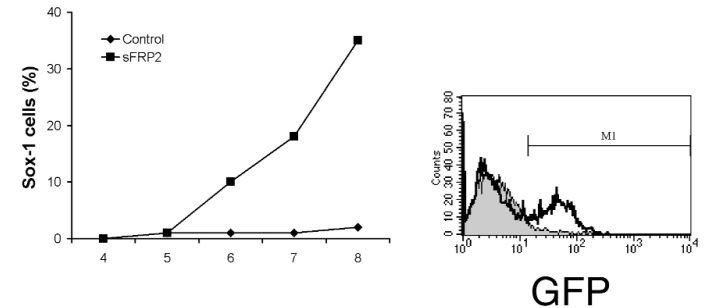

Figure 4. Effect of forced expression of Sfrp2 on differentiation of Sox1GFP ES cells. (A) Sfrp2 expression in 46C parental ES cells (control) and Sfrp2 transfectants. Sfrp2-IRES-pac mRNA was detected by hybridization using a pac probe; Sfrp2 primers for RT-PCR are within the ORF and amplify both endogenous and introduced Sfrp2 sequences. $(\mathrm{B}, \mathrm{C})$ Proportions of Sox $1-\mathrm{GFP}^{+}$cells determined by FACS in control $46 \mathrm{C}$ and Sfrp2-expressing embryoid bodies treated from day 5 to day 8 with $10^{-6} \mathrm{M}$ RA $(\mathrm{B})$ or vehicle $(0.1 \% \mathrm{DMSO})(\mathrm{C})$. Data are representative of three independent experiments. Inserts show flow cytometry profiles at day 8 of control cells (blue) and Sfrp2 cells (green).

gated the effect of lithium chloride, which inhibits GSK-3 and partially mimics Wnt signaling. FACS analyses showed a reduction in the number of Sox $1^{+}$cells generated in response to RA in the presence of lithium (Fig. 5). Collectively, these findings support the interpretation that Sfrp2 promotes neural differentiation of ES cells by blocking Wnt activity.

\section{Discussion}

By subtractive hybridization and differential screening, we isolated genes induced during neural determination of ES cells and expressed in the developing fetal CNS. Functional analysis of candidate genes then identified the Wnt antagonist Sfrp2 as a positive modulator of neural differentiation. This finding was corroborated by evidence that Wnt signaling suppresses neural development. This study thus establishes a methodology for using ES cells directly to identify genes that control pluripotent cell commitment and differentiation. A critical component in this process is efficient cDNA transduction and expression in ES cells through the use of episomal supertransfection.

Gain-of-function expression studies in ES cells are hampered by transcriptional silencing of random integration events. Retroviral expression vectors are rapidly inactivated in ES cells, and randomly integrated eukaryotic promoters also tend to be progressively shut down, often in a mosaic fashion within clonal lines. Even where an introduced expression construct remains fully active in ES cells, it is relatively rare for transcription to be maintained ubiquitously upon differentiation. Therefore, functional assignment by conventional forced expression from integrating vectors requires time-consuming characterization of multiple clones and may still give unclear or misleading data. The use of extrachromosomal vectors overcomes these constraints by permitting the generation of stably and ubiquitously expressing transfectants at high frequency. Routine supertransfection efficiencies of $1 \%$ mean that a single selection plate of $10^{5}$ transfected E14/T cells yields $1 \times 10^{3}$ colonies. With the use of puromycin selection, which rapidly eliminates nontransfected cells, the supertransfectant population is pure within 4 days and is of sufficient size $\left(>10^{6}\right.$ cells) within 14 days to be immediately entered into differentiation assays. Therefore, episomal technology considerably reduces labor and media consumption, and increases the speed and reliability of functional expression screening in ES cells, facilitating parallel evaluation of multiple genes of interest.

The combination of subtractive cloning and functional screening identified Sfrp 2 as a potent promoter of neural differentiation and, more broadly, indicates that the modulation of Wnt activity can inhibit or stimulate neural determination of ES cells. Wnt genes encode a large family of cysteine-rich secreted glycoproteins active in embryonic patterning and cell fate decisions. Wnt proteins induce cellular responses through binding to transmembrane receptors of the frizzled family. Secreted frizzled related proteins (Sfrps) act as extracellular antagonists by binding and sequestering Wnts. To date, five Sfrp family members have been identified in human, mouse, and chicken ${ }^{26,38-41}$. In different systems, Sfrp2 has been demonstrated to be a direct inhibitor of Wnt activity $^{35-37}$.

The impact of Sfrp2 on ES-cell differentiation is profound, mimicking the neural-inducing effect of RA. Conversely, forced expression of Wnt-1 drives nonneural development and suppresses neural differentiation in response to RA. Canonical Wnt signaling entails inactivation of the serine/threonine kinase GSK-3 $\beta$, and subsequent accumulation and nuclear translocation of $\beta$-catenin. In the nucleus, $\beta$-catenin activates Lef/Tcf-mediated transcrip$\operatorname{tion}^{42}$. RA action is mediated through the retinoid receptor proteins RAR and RXR. Intriguingly, evidence has been presented that $\beta$-catenin may interact directly with RAR in a retinoid-dependent manner and decrease activation of T-cell transcription factor $(\mathrm{TCF})^{43}$. This raises the possibility that a major effect of RA in embryoid bodies could be to inhibit Wnt signaling by sequestration of $\beta$-catenin. Thus Sfrp 2 and RA may act respectively as upstream and downstream antagonists of a Wnt- $\beta$-catenin 'antineural' pathway. It is noteworthy that Wnt-3a signaling through Lef1/Tcf1 has been implicated in suppression of neural differentation in the mouse embryo ${ }^{44,45}$ and induction of the mesodermal marker Brachyury ${ }^{46}$.

Neural differentiation in the absence of RA treatment suggests that the effect of Sfrp2 is at the level of primary neural induction. However, Sfrp 2 has been reported to promote clonogenicity and survival of glioma cells ${ }^{47}$ and to modulate programmed cell death (PCD) in the developing chick hindbrain ${ }^{48}$. Therefore, Sfrp 2 may also enhance survival and expansion of neural precursors.

By uncovering the induction and function of Sfrp2, this study has highlighted the importance of Wnt signaling and Wnt antagonism in directing ES-cell differentiation. Further investigations will clarify the pathways and mechanisms involved. Such studies should be expedited by the use of episomal expression technology, which may also be applied to screen candidate regulatory factors in other ES-cell differentiation protocols. 
A

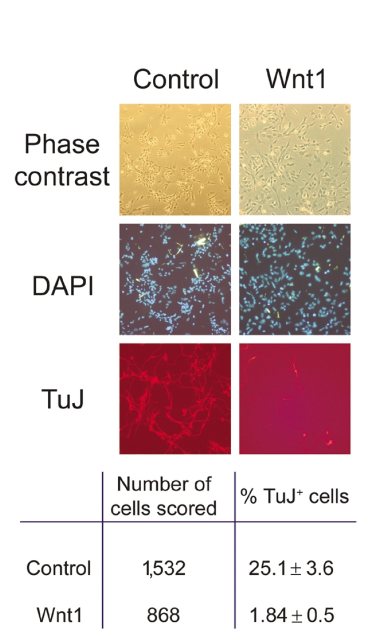

B

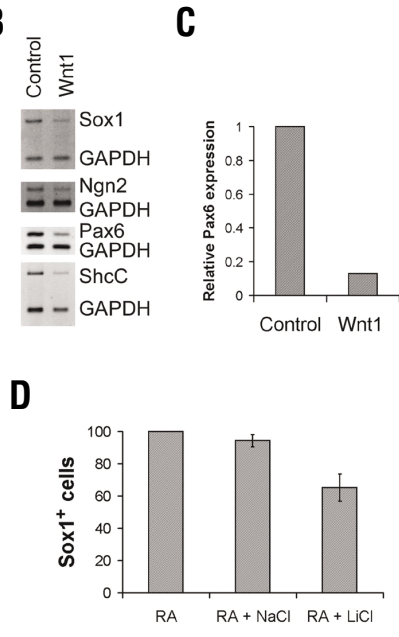

Figure 5. Wnt activity suppresses neural differentiation. (A-C) Embryoid bodies, formed from E14/T cells transfected with pPyHCAGgfp (control) or pPyHCAGwnt1 (Wnt 1), were stimulated from day 4 to day 8 with $10^{-6} \mathrm{M}$ RA and then plated for three days. Cells were immunostained $(A)$ or RNA harvested for analysis by conventional (B) or real-time (C) RT-PCR.

(D) Embryoid bodies formed from 46C Soxgfp cells were treated from day 5 to day 6 with $\mathrm{RA}\left(10^{-6} \mathrm{M}\right), \mathrm{NaCl}(10 \mathrm{mM})$, and $\mathrm{LiCl}(10 \mathrm{mM})$ as indicated, and induction of $\mathrm{GFP}^{+}$cells quantified by flow cytometry. Data are normalized relative to treatment with RA only.

\section{Experimental protocol}

ES-cell culture and differentiation. E14/T cells are derivatives of E14/Tg2a ES cells ${ }^{49}$ that constitutively express polyoma large $\mathrm{T}$ from an integrated cDNA expression cassette, pMGDneo20 (ref. 27). E14/T cells retain normal ES-cell self-renewal and differentiation character. $46 \mathrm{C}$ cells were generated by gene knockin at the Soxl locus to insert the eGFP coding sequence.

ES cells were propagated without feeders in the presence of LIF ${ }^{50}$. Neural differentiation was induced by exposure of embryoid bodies from day 5 to day 8 to all-trans retinoic acid (RA, $\left.10^{-6} \mathrm{M}\right)^{6}$. For terminal differentiation, embryoid bodies at the end of day 8 were plated and allowed to grow out on tissue culture plastic.

For episomal supertransfection, E14/T cells $\left(5 \times 10^{6}\right)$ were electroporated with $20 \mu \mathrm{g}$ of supercoiled plasmid at $200 \mathrm{~V}$ and $960 \mu \mathrm{F}$ in a $0.4 \mathrm{~cm}$ cuvette (Bio-Rad, Hemel Hempstead, UK). A fraction of the cells were plated at $10^{5}$ cells per $9 \mathrm{~cm}$ dish and cultured in the presence of $1.5 \mu \mathrm{g} / \mathrm{ml}$ puromycin for pPyCAGIP constructs or $250 \mu \mathrm{g} / \mathrm{ml}$ of hygromycin for pPyHCAG vectors. Resultant colonies were pooled for differentiation assays. For conventional transfection, $1 \times 10^{7} 46 \mathrm{C}$ cells were electroporated with $100 \mu \mathrm{g}$ of linearized plasmid DNA at $800 \mathrm{~V}$ and $3 \mu \mathrm{F}$, selected in $1 \mu \mathrm{g} / \mathrm{ml}$ of puromycin, and $10-15$ puromycin-resistant colonies pooled and expanded for further analysis.

cDNA library construction and macroarray hybridization. Forward and reverse subtracted CDNA populations were prepared using the PCR-Select kit

(Clontech, Palo Alto, CA). The amplified fragments from the forward subtraction were ligated into pCRII-topo (Invitrogen, Breda, Netherlands). Clone inserts were amplified by PCR from bacterial lysates using adapter sequences from the PCR-Select kit as primers. PCR products were spotted onto Hybond-N+ membrane (Amersham Pharmacia Biotech, Little Chalfont, UK). Subtracted probes were prepared by ${ }^{32} \mathrm{P}$ labeling of $4 \mu \mathrm{l} \mathrm{sam}$ ples of forward and reverse cDNA. Hybridization signals were quantitated using a PhosphorImager (Fuji, Japan).

RNA extraction, dot blot, and RT-PCR. Total RNA and mRNA were extracted using the RNeasy Mini-Kit and Oligotex (Qiagen, West Sussex, UK) respectively. For RNA dot blot, 100 ng of mRNA was directly spotted on Hybond- $\mathrm{N}^{+}$membrane. For RT-PCR, $1 \mu \mathrm{g}$ of total RNA was treated with 1 unit of DNase I for $15 \mathrm{~min}$ at $25^{\circ} \mathrm{C}$. DNase I was inactivated with $25 \mathrm{mM}$ EDTA, pH 8.0, at $65^{\circ} \mathrm{C}$ for $10 \mathrm{~min}$ and chilled on ice. First-strand random-primed cDNA was synthesized using the Superscript II Preamplification System (Gibco BRL, Paisley, UK). Primers and conditions for PCR are provided in Supplementary Table 2 online. Real-time PCR quantitation of Pax-6 transcripts was carried out in a Light Cycler (Roche, Lewes, UK).

In situ hybridization. Mouse embryos were dissected free from extraembryonic membranes, fixed overnight in $4 \%$ PFA-PBS at $4^{\circ} \mathrm{C}$, and dehydrated through a methanol series. Digoxygenin-labeled riboprobes were prepared by antisense and sense in vitro transcription from SP6 and T7 promoters adjacent to the $5^{\prime}$ and $3^{\prime}$ ends, respectively, of cDNA inserts. Embryos were rehydrated and whole-mount in situ hybridization carried out using an InsituPro machine (Anachem, Luton, UK).

Construction of cDNA expression vectors. Primers were designed to amplify full-length ORFs flanked by NotI (5') and XhoI ( $\left.3^{\prime}\right)$ restriction sites. Sequences were amplified from E10.5 mouse embryo cDNA using Expand Long Template PCR (Roche) and cloned in pCRII-topo. After DNA sequence verification, the NotI-XhoI fragments were inserted into the expression vector pPyCAGIP. Wntl cDNA in the pPyHCAG vector was a gift from J. Mason, along with corresponding control GFP expression vector. pPy vectors contain polyoma Ori and can be propagated episomally in cells expressing polyoma $\mathrm{T}$.

Note: Supplementary information is available on the Nature Biotechnology website.

\section{Acknowledgments}

We thank Marios Stavridis for 46C ES cells, and Meng Li, Jenny Nichols, Tom Burdon, and Joe Mee for advice and discussion. This research was supported by the Medical Research Council and the Biotechnology and Biological Sciences Research Council of the UK. J.A. was supported by an INSERM fellowship and a Marie Curie EU Fellowship.

\section{Competing interests statement}

The authors declare competing financial interests: see the Nature Biotechnology website (http://biotech.nature.com) for details.

Received 15 July 2002; accepted 8 October 2002
1. Smith, A. in Stem Cell Biology (eds. Marshak, D. R., Gardner, R. L \& Gottlieb, D.) 205-230 (Cold Spring Harbor Laboratory Press, New York, 2001).

2. Doetschman, T. C., Eistetter, H., Katz, M., Schmidt, W. \& Kemler, R. The in vitro development of blastocyst-derived embryonic stem cell lines: formation of visceral yolk sac, blood islands and myocardium. J. Embryol. Exp. Morphol. 87, 27-45 (1985).

3. Keller, G. M. In vitro differentiation of embryonic stem cells. Curr. Opin. Cell Biol. 7, 862-869 (1995).

4. Smith, A. G. Embryo-derived stem cells: of mice and men. Ann. Rev. Cell Dev. Biol. 17, 435-462 (2001).

5. Fraichard, A. et al. In vitro differentiation of embryonic stem cells into glial cells and functional neurons. J. Cell Sci. 108, 3181-3188 (1995).

6. Bain, G., Kitchens, D., Yao, M., Huettner, J. E. \& Gottlieb, D. I. Embryonic stem cells express neuronal properties in vitro. Dev. Biol. 168, 342-357 (1995).

7. Strubing, C. et al. Differentiation of pluripotent embryonic stem cells into the neuronal lineage in vitro gives rise to mature inhibitory and excitatory neurons. Mech. Dev. 53, 275-287 (1995).

8. Li, M., Pevny, L., Lovell-Badge, R. \& Smith, A. Generation of purified neural precursors from embryonic stem cells by lineage selection. Curr. Biol. 8, 971-974 (1998).
9. Pevny, L. H. \& Lovell-Badge, R. Sox genes find their feet. Curr. Opin. Genet. Dev. 7, 338-344 (1997)

10. Pevny, L. H., Sockanathan, S., Placzek, M. \& Lovell-Badge, R. A role for Sox-1 in neural determination. Development 125, 1967-1978 (1998).

11. Niwa, H., Miyazaki, J. \& Smith, A. G. Quantitative expression of Oct-3/4 defines differentiation, dedifferentiation or self-renewal of ES cells. Nat. Genet. 24, 372-376 (2000).

12. Diatchenko, L. et al. Suppression subtractive hybridization: a method for generating differentially regulated or tissue-specific cDNA probes and libraries. Proc. Natl. Acad. Sci. USA 93, 6025-6030 (1996).

13. Li, M. in Embryonic Stem Cells: Methods and Protocols Vol. 185 (ed. Turksen, K.) 205-215 (Humana Press, Totowa, NJ, 2001).

14. Oulad-Abdelghani, M. et al. Meis2, a novel mouse Pbx-related homeobox gene induced by retinoic acid during differentiation of P19 embryonal carcinoma cells. Dev. Dyn. 210, 173-183 (1997).

15. Leimeister, C., Bach, A. \& Gessler, M. Developmental expression patterns of mouse $s F R P$ genes encoding members of the secreted frizzled-related protein family. Mech. Dev. 75, 29-42 (1998).

16. Nagai, T. et al. The expression of the mouse Zic1, Zic2, and Zic3 gene suggests an essential role for Zic genes in body pattern formation. Dev. Biol. 182, 299-313 (1997). 
17. Uwanogho, D. et al. Embryonic expression of the chicken Sox2, Sox3 and Sox11 genes suggests an interactive role in neuronal development. Mech. Dev. 49, 23-36 (1995).

18. Hargrave, M. et al. Expression of the Sox11 gene in mouse embryos suggests roles in neuronal maturation and epithelio-mesenchymal induction. Dev. Dyn. 210, 79-86 (1997).

19. Cheung, M., Abu-Elmagd, M., Clevers, H. \& Scotting, P. J. Roles of Sox4 in central nervous system development. Mol. Brain Res. 79, 180-191 (2000).

20. Furushima, K., Murata, T., Matsuo, I. \& Aizawa, S. A new murine zinc finger gene. Opr. Mech. Dev. 98, 161-164 (2000).

21. Caubit, X. et al. Vertebrate orthologues of the Drosophila region-specific patterning gene teashirt. Mech. Dev. 91, 445-448 (2000).

22. Cox, G. A., Mahaffey, C. L., Nystuen, A., Letts, V. A. \& Frankel, W. N. The mouse fidgetin gene defines a new role for AAA family proteins in mammalian development. Nat. Genet. 26, 198-202 (2000).

23. Kiss, H. et al. A novel gene containing LIM domains (LIMD1) is located within the common eliminated region 1 (C3CER1) in 3p21.3. Hum. Genet. 105, 552-559 (1999).

24. Zhang, W. J. \& Wu, J.Y. Sip-1, a novel RS domain-containing protein essential for pre-mRNA splicing. Mol. Cell. Biol. 18, 676-684 (1998).

25. Studler, J. M., Glowinski, J. \& Levi-Strauss, M. An abundant mRNA of the embryonic brain persists at a high level in cerebellum, hippocampus and olfactory bulb during adulthood. Eur. J. Neurosci. 5, 614-623 (1993).

26. Rattner, A. et al. A family of secreted proteins contains homology to the cysteinerich ligand-binding domain of frizzled receptors. Proc. Natl. Acad. Sci. USA 94, 2859-2863 (1997).

27. Gassmann, M., Donoho, G. \& Berg, P. Maintenance of an extrachromosomal plasmid vector in mouse embryonic stem cells. Proc. Natl. Acad. Sci. USA 92, 1292-1296 (1995).

28. Camenisch, G. et al. A polyoma-based episomal vector efficiently expresses exogenous genes in mouse embryonic stem cells. Nucleic Acids Res. 24, 3707-3713 (1996)

29. Niwa, H., Burdon, T., Chambers, I. \& Smith, A. G. Self-renewal of pluripotent embry onic stem cells is mediated via activation of STAT3. Genes Dev. 12, 2048-2060 (1998).

30. Niwa, H., Masui, S., Chambers, I., Smith, A. G. \& Miyazaki, J. Phenotypic complementation establishes requirements for specific $\mathrm{POU}$ domain and generic transactivation function of Oct-3/4 in embryonic stem cells. Mol. Cell. Biol. 22, 1526-1536 (2002).

31. Sommer, L., Ma, Q. \& Anderson, D. J. Neurogenins, a novel family of atonal-related bHLH transcription factors, are putative mammalian neuronal determination genes that reveal progenitor cell heterogeneity in the developing CNS and PNS. Mol. Cell. Neurosci. 8, 221-241 (1996).

32. Walther, C. \& Gruss, P. Pax6, a murine paired box gene, is expressed in the developing CNS. Development 113, 1435-1449 (1991).
33. Jostes, B., Walther, C. \& Gruss, P. The murine paired box gene, Pax7, is expressed specifically during the development of the nervous and muscular system. Mech. Dev. 33, 27-37 (1990).

34. Conti, L. et al. Shc signaling in differentiating neural progenitor cells. Nat. Neurosci. 4, 579-586 (2001).

35. Lee, C. S., Buttitta, L. A., May, N. R., Kispert, A. \& Fan, C. M. SHH-N upregulates Sfrp2 to mediate its competitive interaction with WNT1 and WNT4 in the somitic mesoderm. Development 127, 109-118 (2000).

36. Lescher, B., Haenig, B. \& Kispert, A. sFRP-2 is a target of the Wnt-4 signaling pathway in the developing metanephric kidney. Dev. Dyn. 213, 440-451 (1998).

37. Ladher, R. K. et al. Cloning and expression of the Wnt antagonists Sfrp-2 and Frzb during chick development. Dev. Biol. 218, 183-198 (2000).

38. Melkonyan, H. S. et al. SARPs: a family of secreted apoptosis-related proteins. Proc. Natl. Acad. Sci. USA 94, 13636-13641 (1997).

39. Finch, P. W. et al. Purification and molecular cloning of a secreted, Frizzled-related antagonist of Wnt action. Proc. Natl. Acad. Sci. USA 94, 6770-6775 (1997).

40. Salic, A. N., Kroll, K. L., Evans, L. M. \& Kirschner, M. W. Sizzled: a secreted Xwnt8 antagonist expressed in the ventral marginal zone of Xenopus embryos. Development 124, 4739-4748 (1997).

41. Chang, J. T. et al. Cloning and characterization of a secreted frizzled-related protein that is expressed by the retinal pigment epithelium. Hum. Mol. Genet. 8, 575-583 (1999).

42. Yamaguchi, T. P. Heads or tails: Wnts and anterior-posterior patterning. Curr. Biol. 11, R713-R724 (2001)

43. Easwaran, V., Pishvaian, M., Salimuddin \& Byers, S. Cross-regulation of $\beta$-cateninLEF/TCF and retinoid signaling pathways. Curr. Biol. 9, 1415-1418 (1999).

44. Yoshikawa, Y., Fujimori, T., McMahon, A. P. \& Takada, S. Evidence that absence of Wnt-3a signaling promotes neuralization instead of paraxial mesoderm development in the mouse. Dev. Biol. 183, 234-242 (1997).

45. Galceran, J., Farinas, I., Depew, M. J., Clevers, H. \& Grosschedl, R. Wnt3a(-/-)-like phenotype and limb deficiency in Lef1(-/-)Tcf1(-/-) mice. Genes Dev. 13, 709-717 (1999).

46. Yamaguchi, T. P., Takada, S., Yoshikawa, Y., Wu, N. \& McMahon, A. P. T (Brachyury) is a direct target of Wnt3a during paraxial mesoderm specification. Genes Dev. 13, 3185-3190 (1999).

47. Roth, W. et al. Secreted Frizzled-related proteins inhibit motility and promote growth of human malignant glioma cells. Oncogene 19, 4210-4220 (2000).

48. Ellies, D. L., Church, V., Francis-West, P. \& Lumsden, A. The WNT antagonist CSFRP2 modulates programmed cell death in the developing hindbrain Development 127, 5285-5295 (2000).

49. Hooper, M. L., Hardy, K., Handyside, A., Hunter, S. \& Monk, M. HPRT-deficient (Lesch-Nyhan) mouse embryos derived from germline colonization by cultured cells. Nature 326, 292-295 (1987).

50. Smith, A. G. Culture and differentiation of embryonic stem cells. J. Tiss. Cult Methods 13, 89-94 (1991). 